\title{
The Fluctuation in Interest Rate, Exchange Rate and Crude Oil Price and its impact on Stock Market
}

\author{
Dr. Gopal Bihari Saraswat*1, Dr. Madhav Saraswat ${ }^{2}$, Dr. Sanjay Kumar \\ Singh $^{3}$, Dr. Ravi Shekhar Tiwari ${ }^{4}$ \\ ${ }^{1}$ SRM Institute of Science and Technology, Delhi NCR Campus \\ Delhi NCR Campus, Ghaziabad, Uttar Pradesh, India \\ ${ }^{2}$ Ch. Charan Singh University, \\ Meerut, Uttar Pradesh, India. \\ ${ }^{3}$ SRM Institute of Science and Technology, Delhi NCR Campus \\ Delhi NCR Campus, Ghaziabad, Uttar Pradesh, India \\ ${ }^{4}$ SRM Institute of Science and Technology, Delhi NCR Campus \\ Delhi NCR Campus, Ghaziabad, Uttar Pradesh, India \\ ${ }^{1}$ Email: saraswatgopal@gmail.com \\ ${ }^{2}$ E-mail: madhav@rggi.edu.in \\ ${ }^{3}$ E-mail: drsksinghmail@gmail.com \\ ${ }^{4}$ E-mail: ravishet@srmist.edu.in
}

\begin{abstract}
This paper examines relationship of stock market returns with the interest rate, exchange rate and oil price fluctuations. The study used monthly data starting from 2008 to 2018 and used Bai-Perrron multiple break point test to test for structural break in data. Later cointegration test, VAR, VECM and Wald test were used to evaluate causality relationship between the variables. Finally, VDC and IRFs have been applied to judge shocks reverberate through VAR system. The cointegration test result indicated that there is an existence of one long run relationships. Further, VECM revealed a short run causality from exchange rate to Indian stock market returns and the VDC analysis indicated that a positive shock in oil price have a significant effect on Indian stock market returns but the later has a significant effect in deciding interest rate in long run.
\end{abstract}

Keywords: Crude oil price, Generalized Impulse Response, Structural break, Variance Decomposition, Vector Error Correction Model

\footnotetext{
$1 *$ Corresponding author Dr. Gopal Bihari Saraswat is an Assistant Professor at SRM Institute of Science and Technology, Delhi NCR Campus, Ghaziabad, India
} 


\section{Introduction}

Oil price have seen sharp upswing during financial crisis and almost breached the level of $140 \$$ per barrel in year 2008. On the contrary, oil price collapse in year 2014 cause oil price to touch the bottom and price at one time trading at $20 \$$ per barrel in international market. Therefore, volatility in crude oil price always play a havoc for oil importing country like India. Hamilton, (2009) explained three key factors responsible for increase in oil prices (a) strong demand for oil from developing economies especially 'BRIC' (b) low price elasticity of demand (c) low global oil production. The International Energy Outlook, (2017) anticipated that use of liquid fuel for transportation in India to increase by $142 \%$ by year 2040 . Furthermore, according to report BREMMER, (2017) 'The Mixed Fortunes of the BRICS Countries' published in 'Time Magazine', India was home of 8\% of global middle class in 2008 and expected to add another 380 million people by 2030. The growing population in emerging countries and policy measures to alleviate poverty, resulted rise of energy demand in India. Nath Sahu et al., (2014) explained that volatility in microeconomic variables is the repercussion of change in oil prices. When crude oil price shoots up, the general level of price also increased and resulted in low disposable income and decrease in economic demand.

India is the third largest crude oil importer globally and contribute almost $4 \%$ in total share of global crude import according to 'EIA', emerging economy like India, fastest in growth pace and sixth largest economy ${ }^{5}$ in the world, and continue to have larger influence over global economy. Alenzi, (2015) stated three types of risk associated with a country. The crude oil price risk triggers volatility in interest rates, as government of oil importing countries borrow more money to acquire a product and to payout dearer debts in market. Therefore, hike in bond interest rate trigger volatility in exchange rate too Park \& Ratti, (2008). Hence, the present study provides a prominent investigation on the causality of interest rate, exchange rate, crude oil price to stock market.

\section{Literature Review}

There are abundant empirical research studies in past which provide proof of causality between the crude oil price and stock market. Aloui et al., (2012); Bagchi, (2017); Syed A. Basher \& Sadorsky, (2006); Bhar \& Nikolova, (2009); Dutta et al., (2017); Fang \& You, (2014); Ghosh, (2011); Hammoudeh \& Aleisa, (2004); Khan, (2010); Kilian \& Park, (2009); Nath Sahu et al., (2014).Syed A. Basher \& Sadorsky, (2006) study explained the phenomena that there is a positive relationship between the market beta and returns in up and down market. Furthermore, prior studies explained that oil price risk impacts stock market returns and there is conditional relationship between stock market risk and developing country's stock market returns. In addition, Chen et al., (2017); Nath Sahu et al., (2014) also did similar studies. The study of Ceylan et al., (2020) indicated that the crude oil price cause stock market return volatility in long run but has an impartial causality in short run.

Some studies in past found a significant causality among the crude oil price, exchange rate and stock market returns Adebiyi et al., (2010); Syed Abul Basher et al., (2012); Ghosh, (2011); Walid et al., (2011) whereas, study of Majumder \& Nag, (2015) found evidence of bidirectional volatility spillover. Areli Bermudez Delgado et al., (2018) research investigation based on Mexico, revealed that, there is no cointegration or long run relationship among stock market returns, oil price and exchange rates. Further, upswing in exchange rate cause a positive effect on stock market returns.

\footnotetext{
5 As per article "India Is Poised to Become the World's Fifth Largest Economy, But It Can't Stop There", published in 'Forbes Magazine', on December 27, 2017.
} 
Kilian \& Park, (2009) disapprove the conventional studies where oil price is exogeneous and identified the supply and demand shocks cause innovations to the price of oil and stated that 20 percent of long run differences can be explained by these two variables. The study of Fang \& You, (2014) revealed that Indian oil consumption cannot decide the global oil price but volatility in global crude price have negative impact on Indian economy whereas Russian stock market reacts positively on Russian oil-specific supply shocks. Syed Abul Basher et al., (2012) found short term dynamics among macroeconomics variables and emerging economies stock market by using two approaches i.e. (a) standard (b) local projection based. The later approach's impulse response captured more cyclical response than the former one and result suggested that upswing in crude oil prices decreases the stock market return.

Ghosh \& Kanjilal, (2016) investigated oil price impact on Indian stock market by dividing volatility phase 2003 to 2011 in three phases. The study indicated a long run relationship between crude oil prices and stock market returns in phase three (post 2008 crisis). Granger Causality test statistics revealed that oil volatility had impacted the stock market but with no feedback effect.

The studies of Ahmadi et al., (2016); Ftiti et al., (2016) concluded that crude oil price shock has a high exposure to the stock market returns in short and medium term. The exposure in long term is miniscule and market is highly sensitive to oil shock originating from demand shock.

Asghar Anvary Rostamy et al., (2013) concluded that crude oil price, interest rate and exchange rate do have a significant causality on sectoral indices returns, whereas these variables are contrary to other sectoral returns. The study of Alenzi, (2015) based on Gulf Cooperation Council (GCC) concluded that the magnitude of stock market vulnerability to exchange risk is high from the causality between interest rate and oil price risk. The study observed the high exposure in Oman, Qatar and U.A.E. but lower for Saudi Arabia. The study also revealed that interest rate exposure is high on financial firms than nonfinancial firms, but the oil price have shown mixed exposure on the firms.

There is a paucity of research studies in past, on the relationship of Brent crude oil price, exchange rate, interest rate and Indian stock market return. Most of the previous studies were either limited to crude oil price and stock market return, or oil prices, exchange rates and stock market relationship, but none had investigated the impact of price fluctuation of crude, exchange rates and interest rates especially in reference to Indian stock market. The research theme of previous studies moves around developed and emerging economies and results are unable to have linkage to oil price volatility and spillover impact of aforesaid variables on Indian stock market directly.

\section{Research Design}

\subsection{Data Description}

The data of Brent crude price is collected from (EIA) website, though, exchange rate $(₹ / \$)$ and 10-year Government bond yield price data are taken from (investing.com). The daily closing price of 10 -year Government bond yield are the representative of interest rates in this study because the Government borrowing through bonds are considered to be deciding factor of interest rate environment in a country. The EOD (end of day) closing price of Indian stock index Nifty_50 is taken from NSE website (nseindia.com). Later, all daily data of time series variables are transformed into monthly series.

The span of sample data chosen for study is starting from January 2, 2008 to March 30, 2018. This time series data covers the duration since and post global recession. To extract insight from data, Bai-Perron multiple breakpoint test was applied on sample data to identify 
structural break and if structural break found, then a dummy variable is assigned for the break and tested for significance in the model.

\subsection{Methodology}

First, Nifty log returns are calculated and then other variables in the study are transformed into the log. To judge the magnitude of problem, quantum of data and to find data insight from an econometric perspective, all variables are plotted, and descriptive statistics is studied. Later, unit root in time series data of oil price, exchange rate, interest rate and stock index are tested using ADF and PP tests. The Akaike's Information Criterion (AIC), Schwarz Bayesian Criterion (SBC) and another criterion are used to select suitable lag length. Further, if all variables are found stationary then VARs would be estimated with these variables Holden, (1995).

Christopher Sims (1980) study had provided a simple multivariate macroeconomic structure, VARs (Vector Autoregressions) where each variable is explained by its lagged values and the lagged values of all other variables in the system Holden, (1995). The VARs of two or three variables are often unstable and poor predictors of future James H. Stock and Mark W. Watson, (2001) therefore, VARs would be useful as there are four variables under study.

Later, cointegration test is applied to test the VARs model using Johansen \& Juselius (1990) technique to find a long run causality relationship among the variables. If variables are nonstationary but cointegrated, then use of only first differenced variables in a VAR is incorrect. Therefore, Vector ECM (VECM) which is VAR with the addition of a vector cointegration residual must be used for further analysis. Holden, (1995).

VECM (Vector Error Correction Model) is useful in finding long term and short-term relationship. It is also suitable to identify the magnitude and the length of information transmission by an impulse triggered in a series to other connected in the multivariate system. The Generalized Impulse Response (IRF) and Variance Decomposition (VDC) analysis, precise interplay of variables under study and VDC test provide useful information by exploring the degree of exogeneity of the variables.

\section{Empirical Results}

\subsection{Graphical and Descriptive Analysis}

The Fig. 1 shows that Brent crude prices remained highly volatile during global crisis, as it shoots up to $140 \$ /$ barrel in early 2008 and later came down to 38 \$/barrel. The 10 -year Government bond (10yr_Bond) yield price also follows the same trend and phenomenally increased in early crisis period and later come down sharply. This seems that 10yr_Bond and Brent crude prices follow a similar pattern in initial period of study. Exchange rates (Dollar_INR) faced sever volatility in market due to global meltdown and therefore, dollar demand across global markets increased drastically that lead to a decrease in local currency. Hence, because of unprecedent volatility in Brent_Crude,10yr_Bond and Dollar_INR, Indian market (Nifty_50) had to face sharp corrections in 2008. During post crisis (2009-2014) crude prices started to move up but later stabilized between $\$ 105$ to $\$ 115 /$ barrel and similarly, 10yr_Bond yield price stabilized between 8 to 9 percent. On the contrary, Dollar_INR touched high and low as 52/\$ and 69/\$ respectively by 2013-year end and Nifty_50, after touching low as 2675 in early 2009, later recovered and consolidated between 4000 and 6000 level during 2010 to 2014. 
Figure 5.5.1 Global Crude Prices (Brent_Crude), 10 Year Government Bond Price (10yr_Bond), Exchange Rate (Dollar_INR), \& Nifty Daily Closing Prices (Nifty_50) since 2008 to 2018.
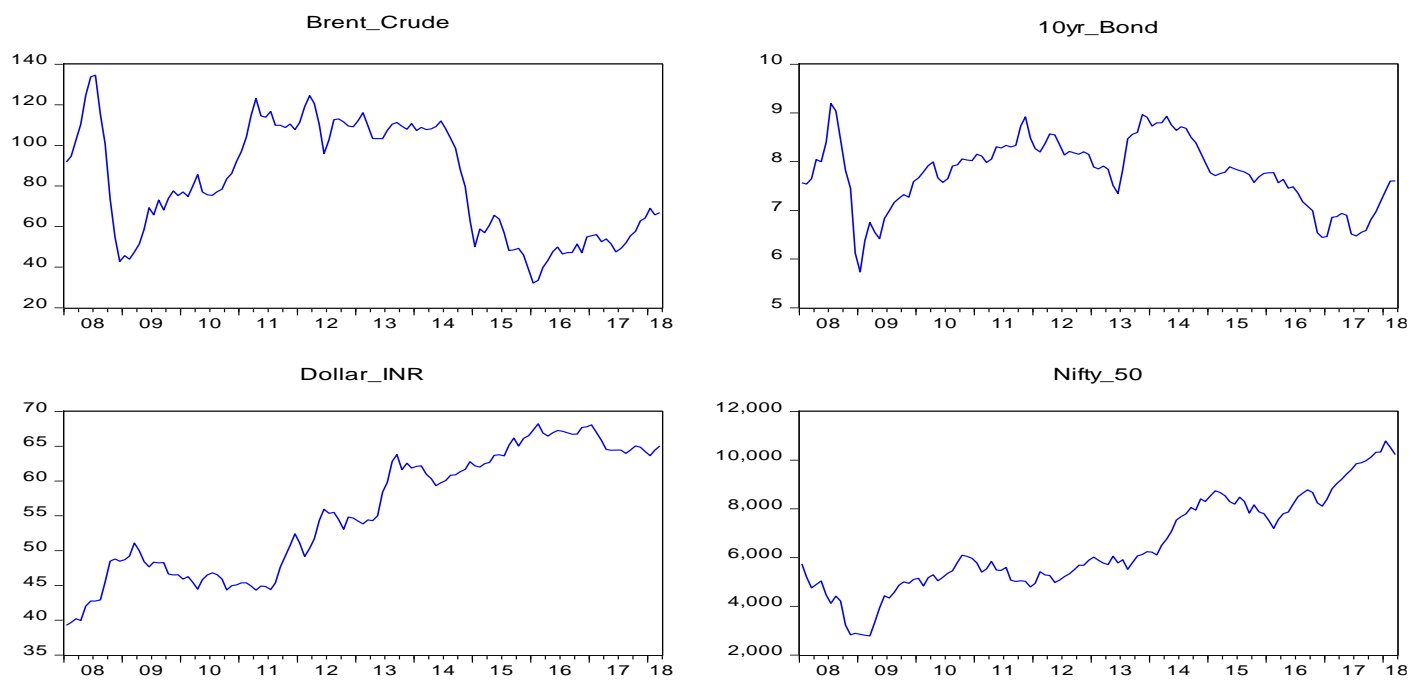

From Table 1, the summary statistics, this can be observed that $\log$ (Brent Crude), Nifty_Return, $\log (10 y$ r_Bond $)$, and $\log ($ Dollar_INR) witnessed a high volatile phase in last decade, as difference in maximum and minimum values are more than $418 \%, 160 \%, 173 \%$, $169 \%$ respectively in aforesaid variables. Furthermore, log values of Brent_Crude and Dollar_INR are highly instable because of high standard deviation. The measure of skewness suggests that the there is no systematical distribution in time series and data is negatively skewed. Moreover, the kurtosis indicates that all series follow platykurtic distribution except Nifty_Return as value of kurtosis is less than 3. Finally, findings from Jarque-Bera test suggests that all variable time series are not normally distributed. But $\log \left(10 \mathrm{yr} \_\right.$Bond $)$is normally distributed at 5 percent level of significance.

Table 1 Summary Statistics: January 2008, to March 2018

\begin{tabular}{lcccc}
\hline \hline & BRENT_CRUDE & _10YR_BOND & DOLLAR_INR & NIFTY_RETURN \\
\hline \hline Mean & 82.17508 & 7.771371 & 55.55801 & 0.004733 \\
Median & 78.41727 & 7.820739 & 55.02187 & 0.015486 \\
Maximum & 134.5648 & 9.191348 & 68.22581 & 0.187121 \\
Minimum & 32.18048 & 5.733273 & 39.27284 & -0.266379 \\
Std. Dev. & 27.59347 & 0.706934 & 8.789554 & 0.053783 \\
Skewness & -0.027415 & -0.432403 & -0.122023 & -0.875115 \\
Kurtosis & 1.574076 & 2.773099 & 1.526061 & 8.580807 \\
& & & & 173.8943 \\
Jarque-Bera & 10.43586 & 4.096794 & 11.43927 & 0.000000 \\
Probability & 0.005419 & 0.128941 & 0.003281 & 0.577375 \\
Sum & 10107.54 & 955.8786 & 6833.635 & 0.350008 \\
Sum Sq. Dev. & 92890.72 & 60.97017 & 9425.264 & 122 \\
Observations & 123 & 123 & 123 & \\
\hline
\end{tabular}




\subsection{Unit Root}

The unit root tests applied in levels are presented in Table 2. It is observed that variables are having unit root i.e., non-stationary except Nifty_Return in both intercept, trend and intercept respectively. Therefore, null hypothesis "the series have a unit root" cannot be rejected at 5 percent level of significance. According to ADF and PP test statistics Nifty_Return is stationary at trend and intercept in levels.

Moreover, the first difference test statistics results of both unit root test statistics suggested that variables are stationary, and the null hypothesis can be rejected at 5 percent level of significance.

Table 1 Results of ADF and PP test (in level)

\begin{tabular}{|c|cc|cc|cc|c|c|}
\hline \multirow{2}{*}{ Variables } & \multicolumn{4}{|c|}{ ADF Test Statistics } & \multicolumn{4}{c|}{ PP Test Statistics } \\
\cline { 2 - 10 } & \multicolumn{2}{|c|}{ Levels } & \multicolumn{2}{c|}{ First Difference } & \multicolumn{2}{c|}{ Levels } & \multicolumn{2}{c|}{ First Difference } \\
& Intercept & $\begin{array}{c}\text { Trend \& } \\
\text { Intercept }\end{array}$ & Intercept & $\begin{array}{c}\text { Trend \& } \\
\text { Intercept }\end{array}$ & Intercept & $\begin{array}{c}\text { Trend \& } \\
\text { Intercept }\end{array}$ & $\begin{array}{c}\text { Intercept } \\
\text { Tntend \& }\end{array}$ \\
\hline LOG(BRENT_CRUDE) & -2.17320 & -2.37736 & -6.92500 & -6.89639 & -1.85577 & -2.05837 & -6.92942 & -6.89891 \\
P-Value & 0.21720 & 0.38940 & 0.00000 & 0.00000 & 0.35220 & 0.56330 & 0.00000 & 0.00000 \\
Log(10YR_BOND)) & -2.36107 & -2.42643 & -8.09913 & -8.06579 & -2.49405 & -2.55608 & -7.65132 & -7.62316 \\
P-Value & 0.15500 & 0.36420 & 0.00000 & 0.00000 & 0.11940 & 0.30120 & 0.00000 & 0.00000 \\
LOG(DOLLAR/INR) & -1.74135 & -2.43004 & -8.14617 & -8.19192 & -1.80872 & -2.00577 & -8.01175 & -8.00183 \\
P-Value & 0.40800 & 0.36240 & 0.00000 & 0.00000 & 0.37480 & 0.59210 & 0.00000 & 0.00000 \\
NIFTY_RETURN & -8.2242 & -8.2137 & -14.6322 & -14.5743 & -8.2435 & -8.2312 & -17.7943 & -17.9401 \\
P-Value & 0.00000 & 0.00000 & 0.00000 & 0.00000 & 0.00000 & 0.00000 & 0.00000 & 0.00000 \\
\hline
\end{tabular}

\subsection{Bai-Perron Multi Breakpoint Test}

The Bai-Perron tests statistics suggests that there is one breakpoint in monthly time series in December, 2008 in data therefore, as mentioned earlier ${ }^{6}$, a dummy variable is introduced (see Table 3) and dummy variable is found significant at 5 percent level. Dummy variable is assigned on the basis of major event timeline during that year, so that these exogeneous variables impact can be investigated during time frame of the study.

\section{Table 1 Breakpoint \& Dummy Variable}

\begin{tabular}{|c|c|}
\hline Breakpoint Month & Dummy Variable \\
\hline December, 2008 & SPCRISIS_2008m12 \\
\hline
\end{tabular}

Notes: SPCRISIS (U.S. Sub Prime Crisis, 2007-08)

${ }^{6}$ See Heading 3.1:

Data Description: Dummy variable is to be assigned for each break. 


\section{Econometric Analysis}

The VAR Model is estimated using $\log$ (Brent_Crude), $\log \left(10 \mathrm{yr} \_B o n d\right), \log ($ Dollar_INR), Nifty_RETURN respectively as endogenous variables and SPCRISIS_2008m12 as exogeneous variable.

\subsection{Optimal Lag Length}

A VAR model may be unstable if mis specified because the chosen lag length is either too large or small then a model may remain mis specified. Therefore, an optimal lag length choice became necessary before testing cointegration technique. The lag order selection was based on the Likelihood ratio (LR) test and information criteria's such as the Final Prediction error (FPE), Akaike's Information Criterion (AIC), SC (Schwarz Information Criterion) and HannaQuinn Information Criterion (HQ). Therefore, according to Table 4 below LR (30.08339) and (AIC (-15.68922) criteria ${ }^{7}$, the recommended lag length is 6. On the contrary, the FPE, SC, LR and HQ criterions recommended different lag order. Consequently, the problem of varying lag length was managed by testing LM test (Lagrange Multiplier residual serial correlation test).

Table 4 Lag Length selection criterion

\begin{tabular}{|ccccccc|}
\hline \hline Lag & LogL & LR & FPE & AIC & SC & HQ \\
\hline \hline 0 & -44.46443 & NA & $2.75 \mathrm{e}-05$ & 0.850253 & 0.946260 & 0.889217 \\
1 & 909.3084 & 1823.881 & $1.97 \mathrm{e}-12$ & -15.60190 & $-15.12187 *$ & $-15.40708^{*}$ \\
2 & 929.8713 & 37.87897 & $1.82 \mathrm{e}-12^{*}$ & -15.68195 & -14.81789 & -15.33128 \\
3 & 944.4914 & 25.90592 & $1.87 \mathrm{e}-12$ & -15.65774 & -14.40965 & -15.15121 \\
4 & 954.9218 & 17.74986 & $2.07 \mathrm{e}-12$ & -15.56003 & -13.92791 & -14.89765 \\
5 & 975.0189 & 32.79004 & $1.94 \mathrm{e}-12$ & -15.63191 & -13.61576 & -14.81367 \\
$\mathbf{6}$ & $\mathbf{9 9 4 . 2 8 5 8}$ & $\mathbf{3 0 . 0 8 3 3 9 *}$ & $\mathbf{1 . 8 6 e}-\mathbf{1 2}$ & $\mathbf{- 1 5 . 6 8 9 2 2} *$ & $\mathbf{- 1 3 . 2 8 9 0 5}$ & $\mathbf{- 1 4 . 7 1 5 1 3}$ \\
7 & 1006.400 & 18.06472 & $2.02 \mathrm{e}-12$ & -15.62105 & -12.83685 & -14.49110 \\
8 & 1019.331 & 18.37661 & $2.18 \mathrm{e}-12$ & -15.56722 & -12.39899 & -14.28141 \\
\hline \hline
\end{tabular}

\section{$5.2 \quad$ LM Test}

The Auto correlation LM Test (see Table 5) rejected the null hypothesis (no serial correlation) at lag 4 and 5 but failed to reject null hypothesis on 6 lags. Therefore, the result supported the previous choice of 6 lags, as recommended by LR and AIC criterions as a suitable lag order for the VAR.

\footnotetext{
7 The lag length criteria of LR (LR Test Statistic), FPE (Final Predication Error), AIC (Akaike Information Criterion), SC (Schwarz Information Criterion) and HQ (Hannan-Quinn Information Criterion) are used to determine the lag length. The lag value lies in most of above-mentioned criterions, the best is lag selection.
} 


\section{Table 5 Autocorrelation Test}

\begin{tabular}{ccc}
\hline \hline Lags & LM-Stat & Prob \\
\hline \hline 1 & 23.83017 & 0.0933 \\
2 & 20.37503 & 0.2038 \\
3 & 24.61200 & 0.0770 \\
4 & 35.11450 & 0.0038 \\
5 & 29.07355 & 0.0234 \\
6 & 19.60279 & 0.2386 \\
\hline \hline
\end{tabular}

\subsection{AR Root Graph}

To test whether VAR model is stable, an AR Root graph plot estimation play a significant role. If all modulus is less than 1 or lie within the range of the unit circle. Figure 2 the AR root graph suggested that all modulus including corner one, are within the unit circle therefore, the VAR is stable and impulse response standard error that will generate on later stage will remain valid. AR root graph also paved the foundation for VECM (if necessary ${ }^{8}$ ), that results of the same will also be valid as all roots are inside the unit circle.

Figure 5.3 AR Root Graph

Inverse Roots of AR Characteristic Polynomial

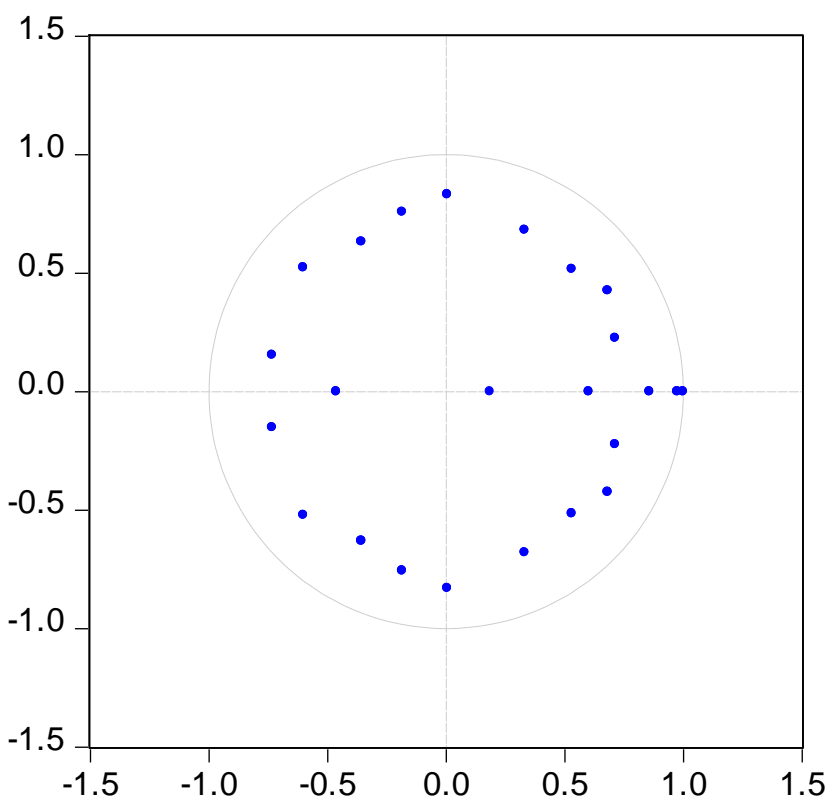

\subsection{Cointegration Test}

The cointegration test statistics results are presented in Table 6 below, the rank test trace and eigenvalue statistics indicated that there are at least 1 cointegration relationship at 5 percent level of significance. The critical values assume that there is no exogenous variables while

${ }^{8}$ If VAR variables are cointegrated then use of VECM is recommended (Holden, 1995). 
calculating cointegration as a result, cointegration calculated for endogenous variables only. Cointegration test statistics calculated in first difference endogenous variables, therefore, the lag length considered one smaller than the recommended lag length criteria of 6 . The null hypothesis (no cointegration or zero cointegration vector) got rejected at 5 percent significance and only 1 cointegration relationships found as a result of cointegration test.

\section{Table 6 Cointegration Test Statistics.}

\begin{tabular}{|c|c|c|c|c|}
\hline \multicolumn{5}{|c|}{$\begin{array}{l}\text { Series: NIFTY_RETURN LOG(BRENT_CRUDE) LOG(_10YR_BOND) } \\
\text { LOG(DOLLAR_INR) } \\
\text { Exogenous series: SPCRISIS_2008M12 } \\
\text { Warning: Critical values assume no exogenous series } \\
\text { Lags interval (in first differences): } 1 \text { to } 5 \\
\text { Unrestricted Cointegration Rank Test (Trace) }\end{array}$} \\
\hline $\begin{array}{l}\text { Hypothesized } \\
\text { No. of CE(s) }\end{array}$ & Eigenvalue & $\begin{array}{c}\text { Trace } \\
\text { Statistic }\end{array}$ & $\begin{array}{c}0.05 \\
\text { Critical Value }\end{array}$ & Prob.** \\
\hline $\begin{array}{c}\text { None } * \\
\text { At most } 1 \\
\text { At most } 2 \\
\text { At most } 3\end{array}$ & $\begin{array}{l}0.233800 \\
0.118659 \\
0.052841 \\
0.009565\end{array}$ & $\begin{array}{l}52.95654 \\
22.06439 \\
7.412304 \\
1.114896\end{array}$ & $\begin{array}{l}47.85613 \\
29.79707 \\
15.49471 \\
3.841466\end{array}$ & $\begin{array}{l}0.0154 \\
0.2950 \\
0.5302 \\
0.2910\end{array}$ \\
\hline \multicolumn{5}{|c|}{$\begin{array}{l}\text { Trace test indicates } 1 \text { cointegrating eqn(s) at the } 0.05 \text { level } \\
* \text { denotes rejection of the hypothesis at the } 0.05 \text { level } \\
* * \text { MacKinnon-Haug-Michelis (1999) p-values } \\
\text { Unrestricted Cointegration Rank Test (Maximum Eigenvalue) }\end{array}$} \\
\hline $\begin{array}{l}\text { Hypothesized } \\
\text { No. of CE(s) }\end{array}$ & Eigenvalue & $\begin{array}{c}\text { Max-Eigen } \\
\text { Statistic }\end{array}$ & $\begin{array}{c}0.05 \\
\text { Critical Value }\end{array}$ & Prob.** \\
\hline $\begin{array}{c}\text { None } * \\
\text { At most } 1 \\
\text { At most } 2 \\
\text { At most } 3\end{array}$ & $\begin{array}{l}0.233800 \\
0.118659 \\
0.052841 \\
0.009565\end{array}$ & $\begin{array}{l}30.89215 \\
14.65208 \\
6.297408 \\
1.114896\end{array}$ & $\begin{array}{l}27.58434 \\
21.13162 \\
14.26460 \\
3.841466\end{array}$ & $\begin{array}{l}0.0181 \\
0.3140 \\
0.5754 \\
0.2910\end{array}$ \\
\hline $\begin{array}{l}\text { Max-eigenvalu } \\
* \text { denotes rejec } \\
* * \text { MacKinnon }\end{array}$ & $\begin{array}{l}\text { indicates } 1 \mathrm{c} \\
\mathrm{f} \text { the hypothe } \\
\text {-Michelis (1) }\end{array}$ & $\begin{array}{l}\text { ating eqn }(\mathrm{s}) \\
\text { he } 0.05 \text { leve } \\
\text { values }\end{array}$ & 0.05 level & \\
\hline
\end{tabular}

\subsection{VECM Model}

The cointegrated variables are related through an error correction term hence, to analyze the long run relationship, VECM is the correct method Holden, (1995). The VECM methodology allows endogenous variables long run behavior to congregate to its long run equilibrium Sandvik \& Følgesvold, (2016).

\section{Table 7 VECM Model Output}

\begin{tabular}{l} 
Vector Error Correction Estimates \\
Sample (adjusted): 2008M08 2018M03 \\
Included observations: 116 after adjustments \\
Standard errors in ( ) \& t-statistics in [ ] \\
\hline Cointegrating Eq: $\quad$ CointEq1 \\
\hline
\end{tabular}




\begin{tabular}{|c|c|c|c|c|}
\hline NIFTY_RETURN(-1) & 1.000000 & & & \\
\hline LOG(BRENT_CRUDE(-1)) & $\begin{array}{r}-0.019489 \\
(0.01872) \\
{[-1.04113]}\end{array}$ & & & \\
\hline LOG(_10YR_BOND(-1)) & $\begin{array}{c}0.021808 \\
(0.06780) \\
{[0.32166]}\end{array}$ & & & \\
\hline LOG(DOLLAR_INR(-1)) & $\begin{array}{r}-0.009987 \\
(0.02841) \\
{[-0.35153]}\end{array}$ & & & \\
\hline $\mathrm{C}$ & 0.072517 & & & \\
\hline Error Correction: & $\begin{array}{l}\text { D(NIFTY_RET } \\
\text { URN) }\end{array}$ & $\begin{array}{l}\text { D(LOG(BREN } \\
\text { T_CRUDE) })\end{array}$ & $\begin{array}{l}\text { D(LOG(_10YR } \\
\text { _BOND)) }\end{array}$ & $\begin{array}{l}\text { D(LOG(DOLL } \\
\text { AR_INR)) }\end{array}$ \\
\hline CointEq1 & $\begin{array}{r}-0.857460 \\
(0.22489) \\
{[-3.81273]}\end{array}$ & $\begin{array}{c}-0.084488 \\
(0.40385) \\
{[-0.20920]}\end{array}$ & $\begin{array}{c}0.086125 \\
(0.13032) \\
{[0.66087]}\end{array}$ & $\begin{array}{r}-0.060141 \\
(0.09663) \\
{[-0.62237]}\end{array}$ \\
\hline D(NIFTY_RETURN(-1)) & $\begin{array}{r}-0.087172 \\
(0.20734) \\
{[-0.42043]}\end{array}$ & $\begin{array}{c}0.049472 \\
(0.37233) \\
{[0.13287]}\end{array}$ & $\begin{array}{r}-0.006859 \\
(0.12015) \\
{[-0.05708]}\end{array}$ & $\begin{array}{c}0.144948 \\
(0.08909) \\
{[1.62698]}\end{array}$ \\
\hline D(NIFTY_RETURN(-2)) & $\begin{array}{c}-0.195804 \\
(0.19028) \\
{[-1.02904]}\end{array}$ & $\begin{array}{c}0.029333 \\
(0.34169) \\
{[0.08585]}\end{array}$ & $\begin{array}{c}0.017868 \\
(0.11026) \\
{[0.16205]}\end{array}$ & $\begin{array}{c}0.159824 \\
(0.08176) \\
{[1.95485]}\end{array}$ \\
\hline D(NIFTY_RETURN(-3)) & $\begin{array}{c}0.122627 \\
(0.16966) \\
{[0.72279]}\end{array}$ & $\begin{array}{c}0.170517 \\
(0.30466) \\
{[0.55969]}\end{array}$ & $\begin{array}{r}-0.037119 \\
(0.09831) \\
{[-0.37757]}\end{array}$ & $\begin{array}{c}0.041909 \\
(0.07290) \\
{[0.57490]}\end{array}$ \\
\hline D(NIFTY_RETURN(-4)) & $\begin{array}{c}0.406173 \\
(0.13234) \\
{[3.06906]}\end{array}$ & $\begin{array}{c}0.223829 \\
(0.23766) \\
{[0.94182]}\end{array}$ & $\begin{array}{r}-0.082616 \\
(0.07669) \\
{[-1.07727]}\end{array}$ & $\begin{array}{r}-0.051609 \\
(0.05687) \\
{[-0.90756]}\end{array}$ \\
\hline D(NIFTY_RETURN(-5)) & $\begin{array}{c}0.118235 \\
(0.08912) \\
{[1.32670]}\end{array}$ & $\begin{array}{c}0.073443 \\
(0.16004) \\
{[0.45892]}\end{array}$ & $\begin{array}{r}-0.162075 \\
(0.05164) \\
{[-3.13839]}\end{array}$ & $\begin{array}{r}-0.005262 \\
(0.03829) \\
{[-0.13740]}\end{array}$ \\
\hline $\begin{array}{c}\text { D(LOG(BRENT_CRUDE(- } \\
1)))\end{array}$ & $\begin{array}{r}0.006636 \\
(0.06055) \\
{[0.10959]}\end{array}$ & $\begin{array}{c}0.273672 \\
(0.10873) \\
{[2.51706]}\end{array}$ & $\begin{array}{c}0.061525 \\
(0.03509) \\
{[1.75358]}\end{array}$ & $\begin{array}{c}0.000512 \\
(0.02602) \\
{[0.01968]}\end{array}$ \\
\hline $\begin{array}{c}\text { D(LOG(BRENT_CRUDE(- } \\
2)))\end{array}$ & $\begin{array}{c}0.019545 \\
(0.06222) \\
{[0.31410]}\end{array}$ & $\begin{array}{c}0.071454 \\
(0.11174) \\
{[0.63947]}\end{array}$ & $\begin{array}{c}0.053629 \\
(0.03606) \\
{[1.48733]}\end{array}$ & $\begin{array}{r}-0.021997 \\
(0.02674) \\
{[-0.82274]}\end{array}$ \\
\hline $\begin{array}{c}\text { D(LOG(BRENT_CRUDE(- } \\
3)))\end{array}$ & $\begin{array}{r}0.048012 \\
(0.06194) \\
{[0.77520]}\end{array}$ & $\begin{array}{c}-0.069359 \\
(0.11122) \\
{[-0.62361]}\end{array}$ & $\begin{array}{r}-0.013137 \\
(0.03589) \\
{[-0.36603]}\end{array}$ & $\begin{array}{c}0.020398 \\
(0.02661) \\
{[0.76648]}\end{array}$ \\
\hline
\end{tabular}




\begin{tabular}{|c|c|c|c|c|}
\hline $\begin{array}{c}\text { D(LOG(BRENT_CRUDE(- } \\
4)))\end{array}$ & $\begin{array}{r}-0.075584 \\
(0.06191) \\
{[-1.22088]}\end{array}$ & $\begin{array}{c}-0.117891 \\
(0.11117) \\
{[-1.06042]}\end{array}$ & $\begin{array}{c}0.037378 \\
(0.03588) \\
{[1.04188]}\end{array}$ & $\begin{array}{r}0.003045 \\
(0.02660) \\
{[0.11446]}\end{array}$ \\
\hline $\begin{array}{c}\text { D(LOG(BRENT_CRUDE(- } \\
5)))\end{array}$ & $\begin{array}{c}0.025787 \\
(0.06143) \\
{[0.41979]}\end{array}$ & $\begin{array}{c}0.050248 \\
(0.11031) \\
{[0.45551]}\end{array}$ & $\begin{array}{r}-0.002226 \\
(0.03560) \\
{[-0.06254]}\end{array}$ & $\begin{array}{r}-0.021840 \\
(0.02639) \\
{[-0.82744]}\end{array}$ \\
\hline D(LOG(_10YR_BOND(-1))) & $\begin{array}{c}-0.081339 \\
(0.16124) \\
{[-0.50445]}\end{array}$ & $\begin{array}{c}-0.539312 \\
(0.28955) \\
{[-1.86256]}\end{array}$ & $\begin{array}{c}0.308756 \\
(0.09344) \\
{[3.30443]}\end{array}$ & $\begin{array}{r}0.060897 \\
(0.06928) \\
{[0.87895]}\end{array}$ \\
\hline D(LOG(_10YR_BOND(-2))) & $\begin{array}{c}0.006554 \\
(0.16914) \\
{[0.03875]}\end{array}$ & $\begin{array}{c}0.583739 \\
(0.30373) \\
{[1.92190]}\end{array}$ & $\begin{array}{c}-0.233523 \\
(0.09801) \\
{[-2.38260]}\end{array}$ & $\begin{array}{r}-0.004521 \\
(0.07268) \\
{[-0.06221]}\end{array}$ \\
\hline D(LOG(_10YR_BOND(-3))) & $\begin{array}{c}0.052320 \\
(0.17969) \\
{[0.29117]}\end{array}$ & $\begin{array}{r}-0.390200 \\
(0.32267) \\
{[-1.20928]}\end{array}$ & $\begin{array}{c}0.036272 \\
(0.10412) \\
{[0.34836]}\end{array}$ & $\begin{array}{r}-0.050399 \\
(0.07721) \\
{[-0.65278]}\end{array}$ \\
\hline D(LOG(_10YR_BOND(-4))) & $\begin{array}{r}-0.201622 \\
(0.16846) \\
{[-1.19682]}\end{array}$ & $\begin{array}{c}0.358037 \\
(0.30252) \\
{[1.18352]}\end{array}$ & $\begin{array}{c}0.023757 \\
(0.09762) \\
{[0.24336]}\end{array}$ & $\begin{array}{c}0.037638 \\
(0.07239) \\
{[0.51997]}\end{array}$ \\
\hline D(LOG(_10YR_BOND(-5))) & $\begin{array}{c}-0.332893 \\
(0.15262) \\
{[-2.18115]}\end{array}$ & $\begin{array}{c}-0.433619 \\
(0.27407) \\
{[-1.58214]}\end{array}$ & $\begin{array}{c}-0.117346 \\
(0.08844) \\
{[-1.32683]}\end{array}$ & $\begin{array}{r}0.104963 \\
(0.06558) \\
{[1.60056]}\end{array}$ \\
\hline D(LOG(DOLLAR_INR(-1))) & $\begin{array}{r}-0.378636 \\
(0.29743) \\
{[-1.27301]}\end{array}$ & $\begin{array}{r}-0.415853 \\
(0.53412) \\
{[-0.77858]}\end{array}$ & $\begin{array}{c}0.258307 \\
(0.17236) \\
{[1.49869]}\end{array}$ & $\begin{array}{r}0.391581 \\
(0.12780) \\
{[3.06399]}\end{array}$ \\
\hline D(LOG(DOLLAR_INR(-2))) & $\begin{array}{r}-0.144096 \\
(0.29970) \\
{[-0.48081]}\end{array}$ & $\begin{array}{c}0.094524 \\
(0.53818) \\
{[0.17564]}\end{array}$ & $\begin{array}{r}-0.007830 \\
(0.17367) \\
{[-0.04509]}\end{array}$ & $\begin{array}{r}-0.093272 \\
(0.12877) \\
{[-0.72432]}\end{array}$ \\
\hline D(LOG(DOLLAR_INR(-3))) & $\begin{array}{c}0.570244 \\
(0.28712) \\
{[1.98611]}\end{array}$ & $\begin{array}{c}0.307642 \\
(0.51559) \\
{[0.59669]}\end{array}$ & $\begin{array}{c}0.001099 \\
(0.16638) \\
{[0.00661]}\end{array}$ & $\begin{array}{r}-0.135443 \\
(0.12337) \\
{[-1.09788]}\end{array}$ \\
\hline D(LOG(DOLLAR_INR(-4))) & $\begin{array}{c}0.742084 \\
(0.28797) \\
{[2.57695]}\end{array}$ & $\begin{array}{c}0.223022 \\
(0.51712) \\
{[0.43128]}\end{array}$ & $\begin{array}{r}-0.038165 \\
(0.16687) \\
{[-0.22871]}\end{array}$ & $\begin{array}{r}-0.325756 \\
(0.12373) \\
{[-2.63270]}\end{array}$ \\
\hline D(LOG(DOLLAR_INR(-5))) & $\begin{array}{r}-0.887369 \\
(0.27805) \\
{[-3.19137]}\end{array}$ & $\begin{array}{r}-0.648645 \\
(0.49931) \\
{[-1.29908]}\end{array}$ & $\begin{array}{c}0.218243 \\
(0.16112) \\
{[1.35450]}\end{array}$ & $\begin{array}{r}0.192210 \\
(0.11947) \\
{[1.60881]}\end{array}$ \\
\hline $\mathrm{C}$ & $\begin{array}{c}0.002833 \\
(0.00448) \\
{[0.63251]}\end{array}$ & $\begin{array}{c}0.004239 \\
(0.00804) \\
{[0.52705]}\end{array}$ & $\begin{array}{c}1.70 \mathrm{E}-05 \\
(0.00260) \\
{[0.00654]}\end{array}$ & $\begin{array}{r}0.002649 \\
(0.00192) \\
{[1.37628]}\end{array}$ \\
\hline SPCRISIS_2008M12 & $\begin{array}{c}-0.065929 \\
(0.02628) \\
{[-2.50903]}\end{array}$ & $\begin{array}{r}-0.184529 \\
(0.04719) \\
{[-3.91066]}\end{array}$ & $\begin{array}{c}-0.052997 \\
(0.01523) \\
{[-3.48054]}\end{array}$ & $\begin{array}{r}0.021888 \\
(0.01129) \\
{[1.93862]}\end{array}$ \\
\hline $\begin{array}{l}\text { R-squared } \\
\text { Adj. R-squared }\end{array}$ & $\begin{array}{l}0.633570 \\
0.546887\end{array}$ & $\begin{array}{l}0.425609 \\
0.289732\end{array}$ & $\begin{array}{l}0.584036 \\
0.485636\end{array}$ & $\begin{array}{l}0.324624 \\
0.164857\end{array}$ \\
\hline
\end{tabular}




\begin{tabular}{lrrrr|} 
Sum sq. resids & 0.164077 & 0.529096 & 0.055095 & 0.030292 \\
S.E. equation & 0.042003 & 0.075427 & 0.024340 & 0.018048 \\
F-statistic & 7.309091 & 3.132302 & 5.935322 & 2.031865 \\
Log likelihood & 215.9418 & 148.0333 & 279.2355 & 313.9292 \\
Akaike AIC & -3.326582 & -2.155747 & -4.417853 & -5.016021 \\
Schwarz SC & -2.780612 & -1.609776 & -3.871883 & -4.470051 \\
Mean dependent & 0.000431 & -0.006027 & -0.001634 & 0.003616 \\
S.D. dependent & 0.062399 & 0.089498 & 0.033938 & 0.019749 \\
& & & \\
\hline Determinant resid covariance (dof adj.) & $1.10 \mathrm{E}-12$ & \\
Determinant resid covariance & $4.54 \mathrm{E}-13$ & & \\
Log likelihood & 990.0456 & & \\
Akaike information criterion & -15.41458 & & \\
Schwarz criterion & -13.13575 & & \\
\hline
\end{tabular}

The result of VECM (see above Table 7) suggested one cointegrating equation with long run normalization relationships in first half of output and the second half consist of error equations with lagged variables. All variables in error correction output are automatically transformed to first differenced and stationery9. The VECM equations representation are given below in equation (1):

D $($ NIFTY_RETURN $)=\mathrm{C}(1) *($ NIFTY_RETURN $(-1)$ -

$0.0194894492935 *$ LOG(BRENT_CRUDE(-1)) + 0.0218082010181*LOG(_10YR_BOND(-

1)) $-0.00998672893739 *$ LOG(DOLLAR_INR(-1)) + 0.072517177624 ) +

C (2)*D(NIFTY_RETURN(-1)) + C(3)*D(NIFTY_RETURN(-2)) +

C(4)*D(NIFTY_RETURN(-3)) + C(5)*D(NIFTY_RETURN(-4)) +

$\mathrm{C}(6) * \mathrm{D}(\mathrm{NIFTY}$ _RETURN(-5)) $+\mathrm{C}(7) * \mathrm{D}($ LOG(BRENT_CRUDE(-1)) $)+$

$\mathrm{C}(8) * \mathrm{D}($ LOG(BRENT_CRUDE(-2)) $)+\mathrm{C}(9) * \mathrm{D}($ LOG(BRENT_CRUDE(-3)) $)+$

$\mathrm{C}(10) * \mathrm{D}($ LOG(BRENT_CRUDE(-4)) $)+\mathrm{C}(11) * \mathrm{D}($ LOG(BRENT_CRUDE(-5)) $)+$

$\mathrm{C}(12) * \mathrm{D}(\mathrm{LOG}($ 10YR_BOND(-1)) $)+\mathrm{C}(13) * \mathrm{D}(\mathrm{LOG}($ 10YR_BOND(-2)) $)+$

$\mathrm{C}(14) * \mathrm{D}(\mathrm{LOG}($ 10YR_BOND(-3))) + C(15)*D(LOG(_10YR_BOND(-4)) ) +

$\mathrm{C}(16) * \mathrm{D}(\mathrm{LOG}($ 10YR_BOND(-5))) + C(17)*D(LOG(DOLLAR_INR(-1))) +

$\mathrm{C}(18) * \mathrm{D}(\mathrm{LOG}(\mathrm{DOLLAR} \mathrm{INR}(-2)))+\mathrm{C}(19) * \mathrm{D}(\mathrm{LOG}(\mathrm{DOLLAR} \mathrm{INR}(-3)))+$

$\mathrm{C}(20) * \mathrm{D}(\mathrm{LOG}(\mathrm{DOLLAR} I \mathrm{INR}(-4)))+\mathrm{C}(21) * \mathrm{D}(\mathrm{LOG}(\mathrm{DOLLAR} I \mathrm{INR}(-5)))+\mathrm{C}(22)+$

C(23)*SPCRISIS_2008M12

\subsubsection{Long Run Causality}

The long run equation (1) system coefficient $\mathrm{C}(1)$ (see Table 8 ) i.e., error correction term is negative but significant (Prob.0.0007<5\%) therefore, findings indicated that there is a long run causality from crude oil price, interest rates and exchange rates to stock market returns.

Table 8 Error Correction Equation Coefficient

\begin{tabular}{|ccccc|}
\hline & Coefficient & Std. Error & t-Statistic & Prob. \\
\hline \hline $\mathrm{C}(1)$ & -0.857460 & 0.224894 & -3.812728 & 0.0002 \\
\hline
\end{tabular}

\subsubsection{Causality in Short Run}

The long run cointegration and causal relationship among the variables drive the present study to further analyze the nature in respect of causality of relationship between variables in

\footnotetext{
${ }^{9}$ As discussed in unit root analysis (Table 4.1.2) variables are stationery after first difference.
} 
short run. Therefore, Wald Test has been applied on error correction equation's lagged coefficient to answer the same. To find out short run causality between stock market return and interest rates and crude price to stock market return, the lagged coefficients from equation (1) were tested using Wald test. The test results (Table 9) suggested that system equation coefficients $C(7)=C(8)=C(9)=C(10)=C(11)=0$ and $C(12)=C(13)=C(14)=$ $C(15)=C(16)=0$ as prob. values for former $(0.8402>5 \%)$ and later $(0.0852>5 \%)$ were insignificant. Hence, there is no short run causality from crude oil price and interest rate to stock market return.

\section{Table 9 Wald Test Statistics of Crude Oil Price and Interest Rate}

\begin{tabular}{|cccc|}
\hline \multicolumn{4}{|c|}{ Wald Test: Equation for Crude Oil Price to } \\
Stock Market \\
$\begin{array}{c}\text { Test } \\
\text { Statistic }\end{array}$ & Value & df & $\begin{array}{c}\text { Probabilit } \\
y\end{array}$ \\
\hline \hline F-statistic & 0.410739 & $(5,93)$ & 0.8402 \\
Chi-square & 2.053693 & 5 & 0.8417 \\
\hline \hline \multicolumn{4}{|c}{$\begin{array}{c}\text { Null Hypothesis: } \\
\text { C(7)=C(8)=C(9)=C(10)=C(11)=0 }\end{array}$} \\
\hline
\end{tabular}

\begin{tabular}{|cccc|}
\hline \multicolumn{3}{|c|}{ Wald Test: Equation for Interest Rate to Stock } \\
Market Return \\
Test Statistic & Value & df & $\begin{array}{c}\text { Probabil } \\
\text { ity }\end{array}$ \\
\hline \hline F-statistic & 2.003904 & $(5,93)$ & 0.0852 \\
Chi-square & 10.01952 & 5 & 0.0747 \\
\hline \hline \multicolumn{4}{|c}{$\begin{array}{c}\text { Null Hypothesis: } \\
C(12)=C(13)=C(14)=C(15)=C(16)=0\end{array}$} \\
\hline
\end{tabular}

On the contrary, Wald test result on lagged equation coefficient $C(17), C(18), C(19), C(20)$ and C(21) of DLOG(Dollar_INR) recommended that $C(17)=C(18)=C(19)=C(20)=$ $C(21) \neq 0$ (see Table 10) hence, there is a short run causality from exchange rates to stock market returns as test result rejected the null hypothesis at 5\% level of significance as (Prob. $0.0004<5 \%$ ). Consequently, stock returns are exposed to short run uncertainty in exchange rates.

\section{Table 10 Wald Test of Exchange Rate}

\begin{tabular}{|c|c|c|c|}
\hline \multicolumn{4}{|c|}{$\begin{array}{l}\text { Wald Test: Equation for Exchange Rate to Stock Market } \\
\text { Return }\end{array}$} \\
\hline Test Statistic & Value & $\mathrm{df}$ & Probability \\
\hline F-statistic & 4.999133 & $(5,93)$ & 0.0004 \\
\hline Chi-square & 24.99566 & 5 & 0.0001 \\
\hline
\end{tabular}

\subsection{Variance Decomposition}

The VDC establishes the relative importance of random impulse that influences the variables in a multivariate system. VDC also measure the shock from itself and other variables. The Table 11 presented below the results of variance decomposition of Nifty_Return, LOG(Brent_Crude), LOG(10yr_Bond), and LOG(Dollar_INR) for a period 10 months.

VDC of Nifty_Return: The VDC results suggested that Nifty_Return played a significant role in explaining itself as the total variation 100 percent in the short run and 74.07 percent in the long run respectively. On the contrary, one standard deviation shock in LOG(Brent_Crude), 
LOG(10yr_Bond), and LOG(Dollar_INR) merely have an impact of 2.54, 1.14 and 13.09 percent respectively on India_Nifty_50 in short run. But in long run, Brent_Crude provides a variation of 2.7 percent to Nifty_Return. Similarly, the innovation shock from LOG(Brent_Crude), LOG(10yr_Bond), and LOG(Dollar_INR) to Nifty_Return remained high as 2.7, 6.44 and 16.77 percent respectively in long run.

VDC of LOG(10yr_Bond): The innovation in LOG(10yr_Bond) explained 94.68 percent in the short run but 67.74 percent in the long run to itself. The innovation shock in exchange rate has only impact of 1.39 percent in short run and 2.87 percent in long run to LOG(10yr_Bond). Nifty_Return is responsible for merely 2.29 percent variation in $10 \mathrm{yr}$ _Bond in short run but in long run, shock in Nifty_Return could have a variation of merely 1.655 percent in LOG(10yr_Bond). Crude price plays an important role in variation in LOG(10yr_Bond) as innovation in crude results 22.97 percent variation in short run and 27.71 percent in long run respectively.

VDC of Dollar_INR: An innovation shock in bond has 5.14 percent shock to exchange rate in long run but merely 2.3 percent in long run. Brent crude do not have any major variation impact on Dollar_INR, in both, short and long run. But Nifty_Return is responsible for a variation of 28.41 percent and 36.71 percent in short and long run respectively. Dollar_INR itself can explain variation of 68 percent and 57.69 percent in short run and long run correspondingly.

VDC of Brent_Crude: The one standard deviation innovation in LOG(10yr_Bond) and LOG(Dollar-INR) could not have any major variation in Brent crude price, both in short and long run. But Brent crude price varies because of shock in itself by 90.74 percent and 88.85 percent in short and long run respectively. Moreover, crude price can have a variation up to 8 percent in short run and 8.04 percent from Nifty_Return in long run.

Result of VDC of all variables suggested that Indian market is strongly endogenous in short and long run and shock in crude price and exchange rate could have variability in Indian market returns especially in long and short run. On the other hand, Nifty returns are exposed to 6.44 percent in long run from interest rate shocks. Crude price largely exposed to its own innovations, but interest rates are largely exposed to crude prices and its own deviations. Stock market innovation too have some short-term impact on interest rate variation, but these shocks are too little to create volatility in interest rates.

Exchange rates are heavily driven by the innovation in stock returns and its own price innovations. Interest rates too contribute to exchange rate volatility in long run. 
Table 11 Variance Decomposition

\begin{tabular}{|c|c|c|c|c|c|}
\hline \multicolumn{6}{|c|}{ Variance Decomposition of NIFTY_RETURN: } \\
\hline Period & S.E. & 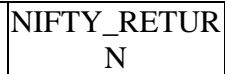 & $\begin{array}{c}\text { LOG(BRENT_CRUD } \\
\text { E) }\end{array}$ & $\begin{array}{c}\text { LOG(_10YR_BON } \\
\text { D) }\end{array}$ & $\begin{array}{c}\text { LOG(DOLLAR_IN } \\
\text { R) }\end{array}$ \\
\hline 1 & 0.042003 & 100.0000 & 0.000000 & 0.000000 & 0.000000 \\
\hline 2 & 0.042987 & 97.78701 & 0.160935 & 0.501050 & 1.551004 \\
\hline 3 & 0.043473 & 95.81681 & 0.567510 & 0.844557 & 2.771124 \\
\hline 4 & 0.044891 & 92.39458 & 1.590214 & 0.886223 & 5.128986 \\
\hline 5 & 0.047812 & 83.20899 & 2.546140 & 1.149319 & 13.09555 \\
\hline 6 & 0.049655 & 77.16121 & 2.368108 & 5.064230 & 15.40645 \\
\hline 7 & 0.050756 & 75.03812 & 2.267048 & 6.215672 & 16.47916 \\
\hline 8 & 0.050824 & 74.91447 & 2.338501 & 6.208997 & 16.53804 \\
\hline 9 & 0.050986 & 74.50911 & 2.571608 & 6.452522 & 16.46676 \\
\hline 10 & 0.051160 & 74.07306 & 2.708293 & 6.448110 & 16.77054 \\
\hline \multicolumn{6}{|c|}{ Variance Decomposition of LOG(BRENT_CRUDE): } \\
\hline Period & S.E. & \begin{tabular}{|c|}
$N_{N}$ \\
\end{tabular} & $\begin{array}{c}\text { LOG(BRENT_CRUD } \\
\text { E) }\end{array}$ & $\begin{array}{c}\text { LOG(_10YR_BON } \\
\text { D) }\end{array}$ & $\begin{array}{c}\text { LOG(DOLLAR_IN } \\
\text { R) }\end{array}$ \\
\hline 1 & 0.075427 & 6.784140 & 93.21586 & 0.000000 & 0.000000 \\
\hline 2 & 0.121865 & 7.565673 & 90.95136 & 1.240270 & 0.242696 \\
\hline 3 & 0.161202 & 7.428762 & 91.03357 & 0.977987 & 0.559682 \\
\hline 4 & 0.191134 & 7.546913 & 90.98607 & 0.974699 & 0.492323 \\
\hline 5 & 0.213195 & 8.046433 & 90.74951 & 0.806533 & 0.397523 \\
\hline 6 & 0.231865 & 8.590231 & 90.22612 & 0.832433 & 0.351213 \\
\hline 7 & 0.249884 & 8.913428 & 89.51460 & 1.096789 & 0.475187 \\
\hline 8 & 0.266329 & 8.617080 & 89.25442 & 1.463524 & 0.664980 \\
\hline 9 & 0.281833 & 8.460317 & 88.97556 & 1.738819 & 0.825303 \\
\hline 10 & 0.295430 & 8.309790 & 88.85234 & 1.942783 & 0.895091 \\
\hline \multicolumn{6}{|c|}{ Variance Decomposition of LOG(_10YR_BOND): } \\
\hline Period & S.E. & \begin{tabular}{|c|} 
NIFTY_RETUR $_{\mathrm{N}}$ \\
\end{tabular} & $\begin{array}{c}\text { LOG(BRENT_CRUD } \\
\text { E) }\end{array}$ & $\begin{array}{c}\text { LOG(_10YR_BON } \\
\text { D) }\end{array}$ & $\begin{array}{c}\text { LOG(DOLLAR_IN } \\
\text { R) }\end{array}$ \\
\hline 1 & 0.024340 & 0.362903 & 4.955890 & 94.68121 & 0.000000 \\
\hline 2 & 0.041658 & 0.920018 & 8.748118 & 89.53261 & 0.799251 \\
\hline 3 & 0.053555 & 2.304341 & 14.80935 & 81.37381 & 1.512504 \\
\hline 4 & 0.062252 & 2.625304 & 18.93949 & 76.87716 & 1.558042 \\
\hline 5 & 0.070820 & 2.293083 & 22.97370 & 73.33563 & 1.397584 \\
\hline 6 & 0.077910 & 1.965757 & 25.55064 & 70.50993 & 1.973670 \\
\hline 7 & 0.083751 & 1.778846 & 26.37094 & 68.88513 & 2.965081 \\
\hline 8 & 0.088571 & 1.900716 & 26.61810 & 68.25231 & 3.228875 \\
\hline 9 & 0.092842 & 1.788386 & 27.05603 & 68.08265 & 3.072933 \\
\hline 10 & 0.097235 & 1.655286 & \begin{tabular}{|l|}
27.71589 \\
\end{tabular} & \begin{tabular}{|l}
67.74948 \\
\end{tabular} & 2.879341 \\
\hline \multicolumn{6}{|c|}{ Variance Decomposition of LOG(DOLLAR_INR): } \\
\hline Period & S.E. & \begin{tabular}{|c|} 
NIFTY_RETUR \\
$\mathrm{N}$ \\
\end{tabular} & $\begin{array}{c}\text { LOG(BRENT_CRUD } \\
\text { E) }\end{array}$ & $\begin{array}{c}\text { LOG(_10YR_BON } \\
\text { D) }\end{array}$ & $\begin{array}{c}\text { LOG(DOLLAR_IN } \\
\text { R) }\end{array}$ \\
\hline 1 & 0.018048 & 34.01707 & 0.718974 & 1.016884 & 64.24707 \\
\hline 2 & 0.029498 & 26.56954 & 0.595447 & 2.169221 & 70.66579 \\
\hline 3 & 0.037636 & 22.37922 & 0.857571 & 2.863573 & 73.89963 \\
\hline 4 & 0.043583 & 23.98601 & 0.728599 & 2.620875 & 72.66452 \\
\hline 5 & 0.047367 & 28.41148 & 0.626703 & 2.365878 & 68.59594 \\
\hline 6 & 0.051508 & 31.68155 & 0.537488 & 2.770459 & 65.01050 \\
\hline 7 & 0.056104 & 33.71024 & 0.496120 & 3.447511 & 62.34613 \\
\hline 8 & 0.060291 & 35.26666 & 0.521665 & 3.972177 & 60.23950 \\
\hline 9 & 0.064369 & 36.21927 & 0.491465 & 4.612991 & 58.67628 \\
\hline 10 & 0.068168 & 36.71469 & 0.438947 & 5.147025 & 57.69934 \\
\hline
\end{tabular}




\subsection{Impulse Response}

Under the IRF's the duration and effect of response of one standard deviation of innovation in 1 residual variable against the other is studied by using Cholesky one standard deviation. The Figure 3 presents the response of one standard deviation innovation in one variable and impulse response to other.

Response to innovation in Nifty_Return: The one positive standard deviation change in Nifty_Return unexpectedly brought itself down in early 3 months but later period Nifty_Return recuperating from zero but fall to negative by 7 months and gradually moves around zero. Innovation in crude price bring nifty return to positive in first 4 months later fall to zero and stabilize there and made it negative. Similarly, interest rate innovation shown a negative change in stock market returns across 10 months. One standard deviation response from exchange rate made nifty returns negative and later to positive unless shock from exchange rate made it negative again by 6 months.

Response to innovation in $\log \left(10 y r_{-}\right.$Bond): The one standard deviation innovation in nifty return brings interest rate upward until 6 months period. Similarly, innovation in crude phenomenally increase interest rates upward over the period. On the other hand, innovation in interest rates itself brought down itself gradually and stabilize in long run. Finally, exchange rate pushed interest rate in beginning but later created volatility.

Response to innovation in LOG(Dollar_INR): The innovation in nifty return made exchange rate to swing upward until 3 months period later gradual decline is witness over the period. Crude price innovation brings a minor increase in exchange rate but stabilize after 5 months. On the other hand, innovation in interest rate brought up exchange rate in early period and keep on increasing over the period. Finally, exchange rate itself made itself volatile in beginning as a response to innovation to itself and later pushed upwards.

Response to innovation in LOG(Brent_Crude): Innovation shock in nifty return lead stable rise in crude prices and same with innovation in crude itself which brought gradual increase in oil prices in early 4 months period. On the contrary, innovation shock in exchange rate made crude price volatile and bring it down to negative in early 3 months period.

The impulse response of variables suggested that an innovation in India_Nifty_50 made itself to reacted negatively but 10yr_Bond, Dollar_INR and Brent_Crude behaved positively. On the other hand, one standard deviation innovation in 10yr_Bond lead India_Nifty_50 to react positively but rest of other variables behaved negatively. Further, shock in exchange rate made itself and Brent_Crude to behave negatively but India_Nifty_50 and 10yr_Bond reacted positively. Finally, exchange rate and crude itself reacted downward to innovation in Brent_Crude but Indian market and 10yr_Bond moved positively. 


\section{Figure 3 Impulse Response of Variables to Stock Market Return}

Response to Cholesky One S.D. Innovations
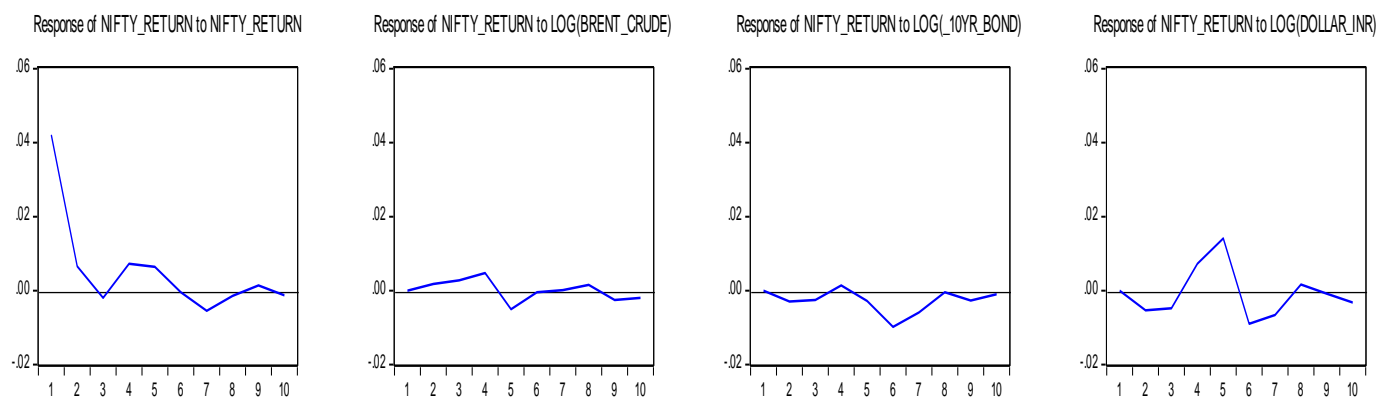

Responsed LOGGBRENT_CRUDE) toNFTY_RETURN

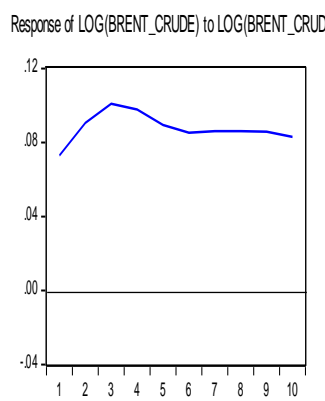

Response of LOG(BRENT_CRUDE) to LOGG_LOYR_BOND)

Response of LOG(BRENT_CRUDE) to LOG(DOLAR_INR)
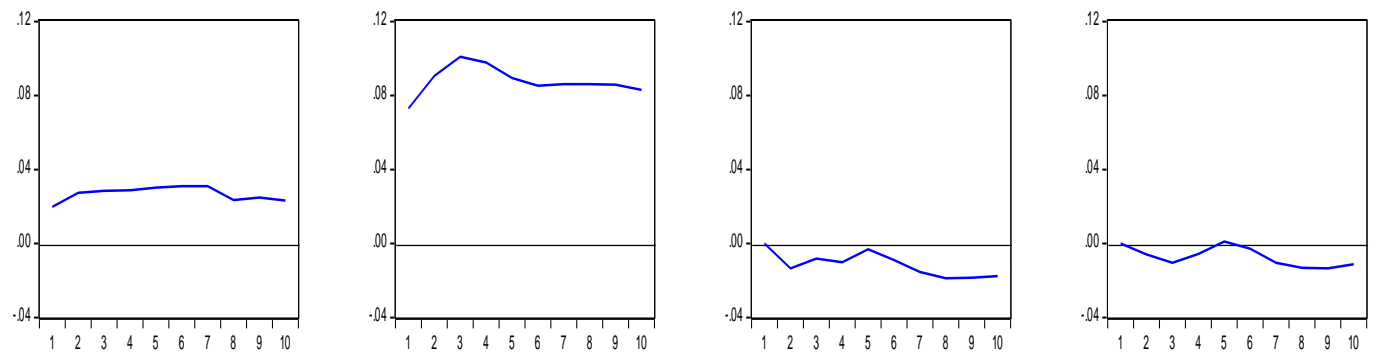

Resporse of LOG(_OYY_BOND) tONFTY_RETURN

Resporse of LOG(_LOYR_BOND) to LOGGBBENT_CRUDE)

Response of LOG(_OYR BOND) I0 LOG( 1OYR BOND)

Response of LOG__LOYR BOND) to LOG_(DOLAR_INR)
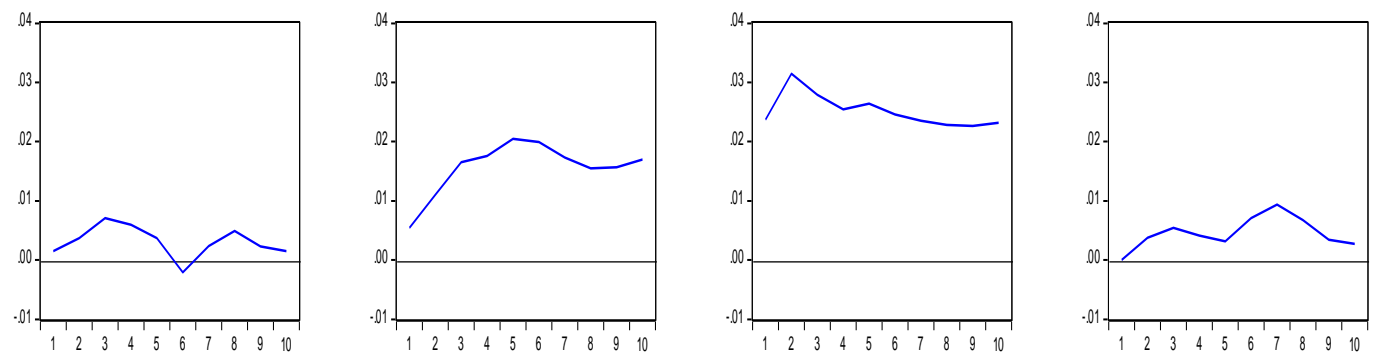

Response of LOG(DOLAR_ INR) to NFFTY _EETURN

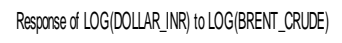

Resporse of LOG(DOLLAR_NRT) to LOG__OYYB_BOND)

Response of LOG(DOLAR_INR) to LOG(DOLAR_INR)
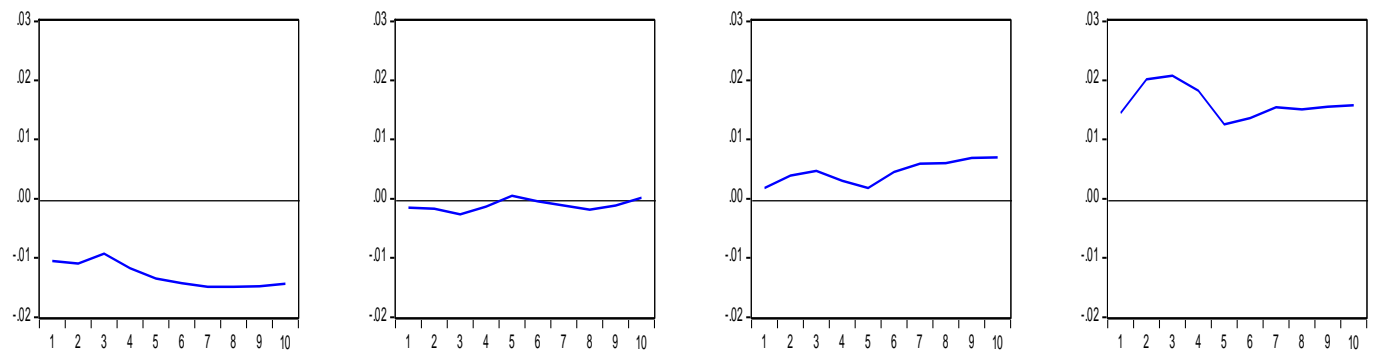

\section{Conclusion}

This paper provides a comprehensive analysis on response of the volatility to stock market returns from the innovation or shocks in crude oil price, exchange rates and interest rates. On aggregate level, findings concludes that there is a long run cointegration relationships: Indian stock market return has a long run causality to crude oil price, exchange rate and interest rate. 
The findings from the test statistics of VECM and the Wald test suggest that there is a significant short run causality from exchange rates to Indian market but not from crude oil price and interest rate. On the contrary, findings indicated a significant long run causality from crude oil price, exchange rates and interest rate to stock market.

The model diagnostic test also suggested that vector error correction model is normal and there is no heteroskedasticity in the model. Further, the VDC analysis suggests that Indian stock market is strongly endogenous. On the other hand, 27.71 percent shock in interest rate can be explained by Brent crude over a period of 10 months. Additionally, Brent crude shock have a spillover impact on Indian stock market in long run and this is found in accordance with the study result of Fang \& You, (2014). Moreover, VECM and VDC study results do suggest that exchange rate shocks have more exposure to Indian stock market in short run than interest rates and Brent crude price, which is contrary to study findings of Nath Sahu et al., (2014). Further, shock in Indian stock market could generate variability of 1.65 percent in interest rate. Finally, Indian macro-economic variables like fluctuation in exchange rate and interest rate could not influence the global crude price but shock in crude price leads to variability in interest rates.

The study compares the empirical evidence of previous researches and develops the literature by studying the relationship in respect to the emerging country's stock market. The study provides a new dimension to policymakers interested in exploring the determinants of Indian stock market and how real economic activity expose to market movements. Further, the study would enable foreign investors keen to invest in Indian market to understand the conditional relationships between the variables.

The future research can be extended to sectoral indices of Indian stock market and empirically test their exposure to Brent crude oil price, interest rate and exchange rate. Finally, the framework presented in this paper could be used to analyze the oil price, exchange rate and interest rate relationship with major oil-importing and oil-exporting countries, such specific study would allow more profound understanding of effect of oil, exchange rate and interest rate shocks on stock markets in these countries. We leave this to the future researchers.

\section{References}

[1] Adebiyi, M. A., Adenuga, A. O., Abeng, M. ., \& Omanukwue, P. . (2010). Oil price shocks, exchange rate and stock market behaviour: Empirical evidence from Nigeria. Central Bank of Nigeria, 1-41. http://www.academia.edu/download/35728338/Adebiyi_Adenuga_Abeng_Omanukwue.pdf

[2] Ahmadi, M., Manera, M., \& Sadeghzadeh, M. (2016). Global oil market and the U.S. stock returns. Energy, 114, 1277-1287. https://doi.org/10.1016/j.energy.2016.08.078

[3] Alenzi, M. (2015). The Impact of Exchange Rate, Interest Rate and Oil Price Fluctuations on Stock Returns Of GCC Listed Companies. University of Plymouth.

[4] Aloui, C., Nguyen, D. K., \& Njeh, H. (2012). Assessing the impacts of oil price fluctuations on stock returns in emerging markets. Economic Modelling, 29(6), 2686-2695. https://doi.org/10.1016/j.econmod.2012.08.010

[5] Areli Bermudez Delgado, N., Bermudez Delgado, E., \& Saucedo, E. (2018). The relationship between oil prices, the stock market and the exchange rate: Evidence from Mexico. North American Journal of Economics and Finance, March, 0-1. https://doi.org/10.1016/j.najef.2018.03.006

[6] Asghar Anvary Rostamy, A., Hosseini, G., \& Bakhshitakanlou, F. (2013). Oil Price, Exchange Rate, Interest Rate, and Market Return Relationships with Industries Stock Returns: Evidence from Iranian Stock Exchange. Research and Applications in Economics, 1(1), 6-12. www.seipub.org/rae

[7] Bagchi, B. (2017). Volatility spillovers between crude oil price and stock markets: evidence from BRIC countries. International Journal of Emerging Markets, 12(2), 352-365. https://doi.org/10.1108/IJoEM04-2015-0077

[8] Basher, Syed A., \& Sadorsky, P. (2006). Oil price risk and emerging stock markets. Global Finance Journal, 17(2), 224-251. https://doi.org/10.1016/j.gfj.2006.04.001

[9] Basher, Syed Abul, Haug, A. A., \& Sadorsky, P. (2012). Oil prices, exchange rates and emerging stock markets. Energy Economics, 34(1), 227-240. https://doi.org/10.1016/j.eneco.2011.10.005

[10] Bhar, R., \& Nikolova, B. (2009). Oil prices and equity returns in the BRIC countries. World Economy, 
32(7), 1036-1054. https://doi.org/10.1111/j.1467-9701.2009.01194.x

[11] BREMMER, I. (2017). The Mixed Fortunes of the BRICS Countries, in 5 Facts. Time Magazine. http://time.com/4923837/brics-summit-xiamen-mixed-fortunes/

[12] Ceylan, R., Ivrendi, M., Shahbaz, M., \& Omay, T. (2020). Oil and stock prices: New evidence from a time varying homogenous panel smooth transition VECM for seven developing countries. International Journal of Finance and Economics, September 2018, 1-16. https://doi.org/10.1002/ijfe.2202

[13] Chen, C. Da, Cheng, C. M., \& Demirer, R. (2017). Oil and stock market momentum. Energy Economics, 68, 151-159. https://doi.org/10.1016/j.eneco.2017.09.025

[14] Dutta, P., Noor, M. H., \& Dutta, A. (2017). Impact of oil volatility shocks on global emerging market stock returns. International Journal of Managerial Finance, 13(5), 578-591. https://doi.org/10.1108/IJMF-03-2017-0039

[15] Fang, C. R., \& You, S. Y. (2014). The impact of oil price shocks on the large emerging countries' stock prices: Evidence from China, India and Russia. International Review of Economics and Finance, 29, 330338. https://doi.org/10.1016/j.iref.2013.06.005

[16] Ftiti, Z., Guesmi, K., \& Abid, I. (2016). Oil price and stock market co-movement: What can we learn from time-scale approaches? International Review of Financial Analysis, 46, 266-280. https://doi.org/10.1016/j.irfa.2015.08.011

[17] Ghosh, S. (2011). Examining crude oil price - Exchange rate nexus for India during the period of extreme oil price volatility. Applied Energy, 88(5), 1886-1889. https://doi.org/10.1016/j.apenergy.2010.10.043

[18] Ghosh, S., \& Kanjilal, K. (2016). Co-movement of international crude oil price and Indian stock market: Evidences from nonlinear cointegration tests. Energy Economics, 53(2014), 111-117. https://doi.org/10.1016/j.eneco.2014.11.002

[19] Hamilton, J. D. (2009). Understanding Crude Oil Prices. NBER Working Paper Series, 14492, 1-44. https://doi.org/10.5547/ISSN0195-6574-EJ-Vol30-No2-9

[20] Hammoudeh, S., \& Aleisa, E. (2004). Dynamic relationships among GCC stock markets and NYMEX oil futures. Contemporary Economic Policy, 22(2), 250-269. https://doi.org/10.1093/cep/byh018

[21] Holden, K. (1995). Vector auto regression modeling and forecasting. Journal of Forecasting, 14(3), 159166. https://doi.org/10.1002/for.3980140302

[22] International Energy Outlook. (2017). International Energy Outlook 2017 Overview. www.eia.go/ieo

[23] James H. Stock and Mark W. Watson. (2001). Vector Autoregressions. Journal of Economic Perspectiv, 15(4), 101-115.

[24] Khan, S. (2010). Crude Oil Price Shocks to Emerging Markets: Evaluating the BRICs Case. Ssrn, 22978, 1-17. https://doi.org/10.2139/ssrn.1617762

[25] Kilian, L., \& Park, C. (2009). The impact of oil price shocks on the U.S. stock market. International Economic Review, 50(4), 1267-1287. https://doi.org/10.1111/j.1468-2354.2009.00568.x

[26] Majumder, S. B., \& Nag, R. N. (2015). Return and volatility spillover between stock price and exchange rate: Indian evidence Sayantan Bandhu Majumder* Ranjanendra Narayan Nag. Int. J. Economics and Business Research, 10(4), 326-340.

[27] Nath Sahu, T., Bandopadhyay, K., \& Mondal, D. (2014). An empirical study on the dynamic relationship between oil prices and Indian stock market. Managerial Finance, 40(2), 200-215. https://doi.org/10.1108/MF-06-2013-0131

[28] Park, J., \& Ratti, R. A. (2008). Oil price shocks and stock markets in the U.S. and 13 European countries. Energy Economics, 30(5), 2587-2608. https://doi.org/10.1016/j.eneco.2008.04.003

[29] Sandvik, A. A., \& Følgesvold, L. R. (2016). Causal relations between stock market returns and macroeconomic variables Cointegration evidence from the Norwegian stock market.

[30] Walid, C., Chaker, A., Masood, O., \& Fry, J. (2011). Stock market volatility and exchange rates in emerging countries: A Markov-state switching approach. Emerging Markets Review, 12(3), 272-292. https://doi.org/10.1016/j.ememar.2011.04.003 